CARTA AL DIRECTOR

\title{
Intentos de suicidio violentos: ¿cuentan los factores sociodemográficos, el perfil clínico o los factores culturales en la repetición del intento?
}

\author{
S. Ruiz-Doblado \\ Unidad de Psiquiatria de Enlace, Hospital de la Merced, Avenida Constitución s/n, 41640 Osuna (Sevilla), \\ España
}

Sr. director:

Hay varios factores que desempeñan un papel en la elección del método de cometer suicidio: la edad, el género, el diagnóstico clínico, la accesibilidad, la aceptabilidad sociocultural, la capacidad letal del método y la imitación de otro comportamiento. Así, aparecen grandes diferencias sociogeográficas y culturales [2]. Por ejemplo, es fácil adquirir armas de fuego en los Estados Unidos, pero esto no es así en Europa. Las estrictas regulaciones referentes a la posesión de armas de fuego en España han significado que este método violento, que era realmente común a principios del siglo XX, se utiliza menos ahora que arrojarse desde un edificio o puente, o ahorcarse. Se ha comunicado que los métodos utilizados por las mujeres son en general más pasivos y menos violentos, siendo el autoenvenenamiento el método más común (Estudio Multicéntrico OMS/EURO sobre el Parasuicidio [1]). En las áreas rurales menos desarrolladas, los métodos no farmacológicos o violentos se utilizan con más frecuencia.

Información tomada del Estudio Epidemiológico de los Intentos de Suicidio en el Hospital de Osuna, un área rural en Sevilla, España, reveló que la frecuencia media de los métodos no farmacológicos (violentos) durante los dos últimos años fue 18,5\% (46 personas con intento de suicidio). Los métodos utilizados más comúnmente eran tragar sustancias cáusticas (12 pacientes, 26,3\%), arrojarse al vacío (12 pacientes, $26,3 \%$ ), ahorcarse (diez pacientes, $21,6 \%$ ), cortarse (diez pacientes, $21,6 \%$ ) y sufrir la embestida de un tren (dos pacientes, 4,2\%). El perfil descriptivo del individuo parasuicida que utiliza un método violento es una persona de mediana edad (proporción varón/mujer de $1,4: 1$ ) con antecedentes psiquiátricos $\mathrm{e}$ intentos anteriores de suicidio, ausencia de autocrítica después de la tentativa y que utiliza métodos accesibles. El espectro de diagnósticos de la CIE-10 [3] era: trastornos de adaptación, neuróticos y somatomorfos, esquizofrenia y otras psicosis, depresión y trastornos mentales orgánicos. No predominaba ninguna categoría nosológica específica.

En comparación con un grupo distribuido al azar de 92 personas con intento de suicidio que utilizaron métodos farmacológicos (pruebas de la $\chi_{2}$, de Kruskal-Wallis y análisis de varianza), no se encontraron diferencias significativas con respecto al género, el grupo de edad, las enfermedades somáticas crónicas, la impulsividad o el grupo diagnóstico de la CIE-10. Por tanto, las variables sociodemográficas básicas consideradas, así como las variables psicológicas/psicopatológicas (rasgo de impulsividad y diagnóstico clínico) no determinaron la elección de método. Sin embargo, parecía haber una asociación con variables como la repetición del intento $(P<0,01)$ o la ausencia de autocrítica posterior $(\mathrm{P}<0,0001)$. Esto señala el hecho 
de que, en nuestra área, el uso de métodos violentos no está tan afectado como se pensaba tradicionalmente por factores como la edad y el género [1, 2] o tan relacionado con el diagnóstico clínico (por ejemplo, el uso de métodos violentos en pacientes con esquizofrenia, depresión mayor o graves). La presencia de intentos anteriores era, sin embargo, esencial. Los pacientes utilizaron estos métodos violentos en el segundo o tercer intento para asegurar su muerte, sin tener en cuenta la edad, el género o el diagnóstico psiquiátrico. Esto coincidía con la persistencia de pensamientos suicidas de los pacientes.

Es posible que la importancia de los factores sociodemográficos y clínicos en la elección del método de suicido se haya sobreestimado; en efecto, según nuestros resultados, la elección del méto- do depende en gran parte de patrones socioculturales, la accesibilidad y el fracaso de los métodos utilizados en intentos anteriores.

\section{BIBLIOGRAFÍA}

1 Bille-Brahe U, Anderson K, Wasserman D, Schmidtke A, Bjerke T, Creper R, et al. The WHO-EURO Multicentre Study: risk of parasuicide and the comparability of the areas under study. Crisis 1996; 17: 32-42.

2 Saiz-Martínez PA, González MP, Cocaña I, Bobes J. Epidemiología. In: Bobes J, González-Seijó JC, SáizMartínez PA, Eds. Prevención de las conductas suicidas y parasuicidas. Barcelona: Masson; 1997. p. 21-34.

3 World Health Organization (WHO). The multiaxial presentation of ICD-10 for use in adult psychiatry. Geneve: WHO; 1996. 